\title{
EFEITOS DA SIMULAÇÃO DE DERIVA DE CLOMAZONE EM PLANTAS DE LARANJEIRA 'HAMLIN' 1
}

\author{
PAULO CÉSAR TIMOSSI² \& PEDRO LUÍS DA C.A. ALVES ${ }^{3}$
}

\begin{abstract}
RESUMO Em trabalho realizado em 1998, no município de Taiúva-SP, objetivou-se avaliar o efeito da simulação da deriva de doses crescentes, até atingir a recomendada comercialmente, de clomazone, em duas formulações, e de clomazone em mistura com ametryne, em laranjeira-'Hamlin' com frutos com 2 a $4 \mathrm{~cm}$ de diâmetro. O delineamento experimental utilizado foi o de blocos casualizados, com 15 tratamentos, em 3 repetições. As avaliações basearam-se em possíveis alterações morfofisiológicas das plantas, com determinações do teor de clorofila total nas folhas, porcentagem de abortamento de frutos, além de análise tecnológica dos frutos. Concluiu-se que a dose comercial de clomazone isolado e em mistura com ametryne a 50 e $100 \%$ da dose resultou na formação de manchas cloróticas e/ ou necróticas na casca do fruto e causou mortalidade de ramos que se encontravam em crescimento vegetativo, não acarretando qualquer alteração qualitativa do suco.
\end{abstract}

Termos de indexação: herbicida, deriva, fitointoxicação.

\section{EFFECTS OF CLOMAZONE DRIFT SIMULATION ON HAMLIN ORANGE FRUITS}

\begin{abstract}
The experiment aimed to evaluate the clomazone (alone or in mixture with atrazine) drift off simulation, on quantitative and qualitative aspects of the sweet orange production when occurred at developmental plant stages of fruit growing (fruits of 2 until $4 \mathrm{~cm}$ diameter). Crescents doses, until commercial rate, of clomazone, in two formulations, and in mixture with ametryne were tested. The experimental design was completely randomized blocks, with 15 treatments and 3 replications. It was evaluate: fruit morphological feature, fruits abortion and leaf chlorophyll content. Clomazone alone at of commercial rate and in mixture with ametryne at 50 and $100 \%$ promoted clorotic and/or necrotic spots in the fruit skin and promoted the death of new sprouting stems. There were no qualitative effects on juice.
\end{abstract}

Index terms: herbicides, drift, phytointoxication.

\section{INTRODUÇÃO}

Apesar das grandes produções obtidas pelo setor citrícola nos últimos anos, esse tem apresentado uma base tecnológica baixa, uma vez que ele não se tem mostrado muito rentável e, conseqüentemente, não proporcionado o devido retorno de investimentos na cultura.

Na região citrícola do Estado de São Paulo, a erradicação de pomares coincidiu principalmente com a expansão das áreas de plantio de cana-de-açúcar, fazendo com que uma cultura fosse trocada por outra ou se tornassem freqüentemente vizinhas.

O setor sucroalcooleiro vem procurando otimizar suas aplicações de defensivos como uma forma de reduzir despesas e, com este objetivo, aplicações aéreas que vêm sendo muito utilizadas para herbicidas e maturadores, mas aumentam o risco de deriva desses produtos em culturas que não eram alvo (Gelmini, 1988). Ao realizar a escolha de um determinado herbicida, devese avaliar os riscos e benefícios do uso do produto, considerando-se a forma de uso, a importância econômica e a presença de culturas vizinhas suscetíveis (Auch \& Arnold, 1978). O objetivo da aplicação de um herbicida é colocar a quantidade certa de ingrediente ativo no alvo desejado, com a máxima eficiência e da maneira mais econômica possível, sem afetar o meio ambiente (Durigan, 1989).

Segundo Matuo (1990), a aplicação de defensivos, principalmente a aérea, quando feita na presença de ventos com velocidade acima de $5 \mathrm{~km} / \mathrm{h}$, pode provocar o carregamento de partículas menores do produto por longas distâncias (deriva), provocando danos, em alguns casos, às culturas ou plantas que não eram seus alvos. Depreende-se, assim, que o herbicida deve atingir de modo efetivo a planta daninha a ser controlada, uma vez que a ocorrência de deriva, além de reduzir a eficiência da aplicação (Gelmini, 1988), coloca em risco culturas vizinhas suscetíveis. Gotas pequenas podem ser transportadas em condições variáveis de vento e luminosidade, particularmente em condições de inversão térmica, atingindo assim áreas agrícolas adjacentes, sendo possível verificar sintomas de injúrias causados por deriva de herbicidas a quilômetros de distância do local de aplicação (Yates et al., 1978).

Para Bayley \& Kapusta (1993), a iminente possibilidade de ocorrência de injúrias em culturas, devido à deposição de herbicidas em áreas "não alvo", tem levado à condução de várias pesquisas envolvendo o conceito de "deriva simulada", principalmente em culturas nas quais as aplicações são realizadas com equipamento aéreo.

A ocorrência de riscos de deriva de produtos seletivos

1 Trabalho $n^{\circ}$ 110/2000. Recebido: 26/06/2000. Aceito para publicação: 25/06/2001.

2 Eng. Agr., Mestrando em Produção Vegetal pela FCAVJ-UNESP

3 Prof. Assist. Doutor do Depto. de Biologia Aplicada à Agropecuária da FCAVJ-UNESP. Rod. de acesso Prof. Paulo D. Castellane, s/n,

CEP 14884-900, Jaboticabal, SP. E-mail: plalves@fcav.unesp.br 
à cultura de cana-de-açúcar, em culturas suscetíveis como a de citros, vem causando sérios problemas devido a pressupostos erros na tecnologia de aplicação.

Em virtude do relatado, este trabalho objetivou avaliar o efeito da deriva simulada de clomazone, em duas formulações, e de clomazone em mistura com ametryne, herbicidas esses normalmente utilizados no controle de plantas daninhas em pré e pós-emergência na cultura da cana-de-açúcar, sobre características quantitativas e qualitativas de frutos de laranjeira (Citrus sinensis) cv. Hamlin.

\section{MATERIAL E MÉTODOS}

$\mathrm{O}$ experimento foi instalado em área de produção de laranja (Citrus sinensis L. Osbeck) cv. Hamlin durante o ano agrícola de 1998, no município de Taiúva-SP. As plantas de laranjeira utilizadas na instalação do ensaio apresentavam-se vigorosas, com frutos, e emitindo novos ramos vegetativos, com quatorze anos e condições fitossanitárias normais.

Os tratamentos experimentais constaram da aplicação de doses crescentes de clomazone, isolado ou em mistura com ametryne (até que essas atingissem a dose recomendada comercialmente para o controle de plantas daninhas), no estágio de desenvolvimento de frutos, com 2 a $4 \mathrm{~cm}$ de diâmetro. A descrição dos tratamentos experimentais utilizados encontra-se na Tabela 1.

A aplicação dos herbicidas foi realizada com pulverizador costal à pressão constante (ar comprimido), munido de um bico XR11002 e regulado para um gasto de volume de calda de $200 \mathrm{~L} /$ ha. Essa aplicação foi realizada a um metro de distância das árvores, direcionada para a porção mediana dessas, resultando em uma área tratada de $0,45 \mathrm{~m}^{2}$ por aplicação.

As parcelas experimentais constaram de faixas de aplicação $\left(0,45 \mathrm{~m}^{2}\right)$, sendo que foram aplicadas três faixas em faces opostas de uma mesma planta, correspondendo, cada qual, a uma repetição do tratamento. Como testemunha, foram consideradas três faixas $\left(0,45 \mathrm{~m}^{2}\right)$ por planta não tratada. $\mathrm{O}$ experimento foi instalado no campo segundo o delineamento experimental de blocos ao acaso, com 15 tratamentos e três repetições.

Delimitada a área da parcela, foram marcados com tinta branca dez frutos por faixa tratada, nos quais foram avaliados os efeitos dos tratamentos. Esses efeitos foram avaliados aos 15; 30; 60; 90 e 120 dias após aplicação (DAA), realizando cálculo da porcentagem de abortamento acumulado aos 120 DAA. Nestas mesmas ocasiões, foram feitas determinações dos teores relativos de clorofila total em folhas tomadas ao acaso no terço final dos ramos e no terço médio das folhas nas faixas tratadas, utilizando um clorofilômetro manual (Minolta, mod. SPAD 502). Ainda, foram acompanhadas quaisquer alterações morfofisiológicas nas folhas e frutos resultantes dos tratamentos experimentais.

Aos 90 DAA, foram efetuadas medições do diâmetro transversal de três frutos remanescentes na faixa tratada, utilizando, para tanto, um paquímetro de precisão. Aos 180DAA, por ocasião da colheita dos frutos, esses foram submetidos a análises tecnológicas, seguindo padrões industriais, que foram: diâmetro longitudinal e transversal dos frutos, teor de sólidos solúveis totais ( ${ }^{\circ}$ Brix), acidez titulável e índice de maturação ("ratio"), segundo Tressler \& Joslyn (1961), e porcentagem de suco realizados com cinco frutos remanescentes da área tratada, tomados ao acaso. Os dados obtidos para ${ }^{\circ} \mathrm{Brix}$ foram corrigidos para temperatura padrão de $20^{\circ} \mathrm{C}$.

Os resultados obtidos foram submetidos à análise de variância pelo teste $\mathrm{F}$ e as médias foram comparadas pelo teste de Tukey, ao nível de 5\% de probabilidade. Para fins de normalização, os resultados expressos em porcentagem foram transformados para arc senÖx antes de serem submetidos à análise de variância.

\section{RESULTADOS E DISCUSSÃO}

A simulação da deriva de clomazone isolado ou em mistura com ametryne em frutos de laranjeira-'Hamlin', com 2 a 4 centímetros de diâmetro, não causou abortamento significativo de frutos até os 30 DAA (Tabela 2). Aos 45 e 90 DAA observouse que a aplicação da dose de $50 \%$ de clomazone isolado resultou em maior taxa de aborto do que os dos demais tratamentos, incluindo a testemunha. Contudo, aos 120 DAA, não mais se observou diferença entre os efeitos dos tratamentos, inclusive quando se calculou porcentagem acumulada de abortamento.

A análise do teor relativo de clorofila total nas folhas demonstrou que nenhum dos tratamentos alterou o seu conteúdo relativo dos 15 aos $120 \mathrm{DAA}$, com média de 62 unidades relativas (UR) nas folhas submetidas aos tratamentos, contra 63 UR nas folhas da testemunha, indicando que o fluxo do produto absorvido ocorreu em direção aos frutos em desenvolvimento, corroborando com as observações visuais. Como as folhas que receberam o produto, se encontram totalmente expandidas, o produto não exerceu seu modo de ação, ou seja, não atuou inibindo a síntese de clorofilas e carotenóides (Rodrigues \& Almeida, 1998), e as folhas simplesmente atuaram como observadoras, translocando o produto para o dreno atuante, seguindo o fluxo transpiracional.

Quanto à análise tecnológica dos frutos (Tabela 3), verificou-se aos 90 DAA que nenhum dos tratamentos testados resultou em frutos de menor diâmetro quando comparados com a testemunha. Por ocasião da colheita, aos 180DAA, observouse baixa redução no diâmetro dos frutos para as formulações utilizadas em relação à testemunha. Ainda, constatou-se menor diâmetro obtido para a média de frutos submetidos ao tratamento com clomazone em mistura com ametryne quando se aplicou $50 \%$ da dose (CA-50).

Aos 100DAA (Figura 01-A), verificou-se visualmente que frutos tratados com a maior dose de clomazone isolado e em mistura com ametryne, a 50\% e 100\% (CA-50 e CA-100) da dose comercial, apresentavam manchas necróticas, sendo que a formação dessas foram mais perceptíveis nos tratamentos de clomazone em mistura. A formulação microencapsulada não causou formação dessas manchas nos frutos. Através de dados analisados para o índice de maturação (Ratio), observa-se que a testemunha, mesmo sem diferir dos demais tratamentos, com exceção do tratamento C-12,5, apresenta-se abaixo dos valores obtidos pelos demais.

Após aplicação da maior dose de clomazone e as duas maiores em mistura com ametryne, quando a planta se encontrava em pleno crescimento vegetativo, ou seja, com emissão de novos 
TABELA 1 - Descrição dos tratamentos experimentais, com as respectivas doses dos ingredientes ativos (i.a.) e dos produtos comerciais, e suas correspondências com a concentração porcentual, aplicados em laranjeira-Hamlin (\%v/v). Taiúva, 1998

\begin{tabular}{|c|c|c|c|c|}
\hline \multirow{2}{*}{$\mathrm{N}^{\mathrm{o}}$} & \multirow{2}{*}{ Tratamentos } & \multicolumn{2}{|c|}{ Dosagem } & \multirow{2}{*}{$\begin{array}{c}\text { Concentração } \\
(\% \mathrm{v} / \mathrm{v})\end{array}$} \\
\hline & & i.a. $(\mathrm{kg} / \mathrm{ha})$ & p.c. (kg ou L/ha) & \\
\hline 1 & Clomazone $* \mathrm{C}-3,12$ & 0,031 & 0,062 & 3,12 \\
\hline 2 & Clom azone $-\mathrm{C}-6,25$ & 0,062 & 0,125 & 6,25 \\
\hline 3 & Clom azone $-\mathrm{C}-12,5$ & 0,125 & 0,250 & 12,50 \\
\hline 4 & Clomazone $-\mathrm{C}-25$ & 0,250 & 0,500 & 25,00 \\
\hline 5 & Clomazone $-\mathrm{C}-50$ & 0,500 & 1,000 & 50,00 \\
\hline 6 & Clom azone $-\mathrm{C}-100$ & 1,000 & 2,000 & 100,00 \\
\hline 7 & Clom azone+ametrina $* * \mathrm{CA}-1,06$ & $0,015+0,023$ & 0,078 & 1,06 \\
\hline 8 & Clomazone + ametrina $-\mathrm{CA}-3,12$ & $0,031+0,047$ & 0,156 & 3,12 \\
\hline 9 & Clomazone + ametrina $-\mathrm{CA}-6,25$ & $0,062+0,094$ & 0,312 & 6,25 \\
\hline 10 & Clomazone + ametrina $-\mathrm{CA}-12,5$ & $0,125+0,187$ & 0,625 & 12,50 \\
\hline 11 & Clomazone + ametrina $-\mathrm{CA}-25$ & $0,250+0,375$ & 1,250 & 25,00 \\
\hline 12 & Clomazone + ametrina - C A -50 & $0,500+0,750$ & 2,500 & 50,00 \\
\hline 13 & Clomazone + ametrina $-\mathrm{CA}-100$ & $1000+1500$ & 5,000 & 100,00 \\
\hline 14 & Clomazone***-CM & 1000 & 2,770 & 100,00 \\
\hline 15 & Testemunha & 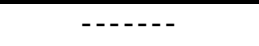 & ----- & ------ \\
\hline
\end{tabular}

Produto comercial:* Gamit; ** Sinerge; *** Gamit microencapsulado

TABELA 2 - Porcentagens de frutos abortados no decorrer do período experimental, em função dos tratamentos aplicados em frutos de 2 a 4 cm de diâmetro. Taiúva, 1998

\begin{tabular}{|c|c|c|c|c|c|c|c|c|}
\hline \multirow{2}{*}{ Tratamentos } & \multicolumn{6}{|c|}{ Porcentagem de abortamento de frutos } & \multicolumn{2}{|c|}{ Número final } \\
\hline & $15 \mathrm{DAA}$ & $30 \mathrm{DAA}$ & $45 \mathrm{DAA}$ & $60 \mathrm{DAA}$ & $90 \mathrm{DAA}$ & $120 \mathrm{DAA}$ & Orig. & Transf. \\
\hline $\mathrm{C}-3,12$ & $0,0 \mathrm{~A}$ & $0,0 \mathrm{~A}$ & $0,0 \mathrm{~B}$ & 0 & $0,0 \mathrm{~B}$ & $0,0 \mathrm{~A}$ & 0 & $0,0 \mathrm{~A}$ \\
\hline$C-12,5$ & $0,0 \mathrm{~A}$ & $0,0 \mathrm{~A}$ & $0,0 \mathrm{~B}$ & 0 & $0,0 \mathrm{~B}$ & $0,0 \mathrm{~A}$ & 0 & $0,0 \mathrm{~A}$ \\
\hline$C-25$ & $0,0 \mathrm{~A}$ & $0,0 \mathrm{~A}$ & $0,0 \mathrm{~B}$ & 0 & $0,0 \mathrm{~B}$ & $0,0 \mathrm{~A}$ & 0 & $0,0 \mathrm{~A}$ \\
\hline$C-100$ & $0,0 \mathrm{~A}$ & $0,0 \mathrm{~A}$ & $0,0 \mathrm{~B}$ & 0 & $0,0 \mathrm{~B}$ & $0,0 \mathrm{~A}$ & 0 & $0,0 \mathrm{~A}$ \\
\hline $\mathrm{CA}-1,06$ & $0,0 \mathrm{~A}$ & $3,3 \mathrm{~A}$ & $0,0 \mathrm{~B}$ & 0 & $0,0 \mathrm{~B}$ & $0,0 \mathrm{~A}$ & 1 & $1,9 \mathrm{~A}$ \\
\hline $\mathrm{CA}-3,12$ & $0,0 \mathrm{~A}$ & $0,0 \mathrm{~A}$ & $0,0 \mathrm{~B}$ & 0 & $0,0 \mathrm{~B}$ & $0,0 \mathrm{~A}$ & 0 & $0,0 \mathrm{~A}$ \\
\hline$C A-6,25$ & $0,0 \mathrm{~A}$ & $0,0 \mathrm{~A}$ & $0,0 \mathrm{~B}$ & 0 & $0,0 \mathrm{~B}$ & $0,0 \mathrm{~A}$ & 0 & $0,0 \mathrm{~A}$ \\
\hline$C A-100$ & $0,0 \mathrm{~A}$ & $0,0 \mathrm{~A}$ & $0,0 \mathrm{~B}$ & 0 & $0,0 \mathrm{~B}$ & $0,0 \mathrm{~A}$ & 0 & $0,0 \mathrm{~A}$ \\
\hline $\mathrm{CM}$ & $0,0 \mathrm{~A}$ & $0,0 \mathrm{~A}$ & $0,0 \mathrm{~B}$ & 0 & $0,0 \mathrm{~B}$ & $0,0 \mathrm{~A}$ & 0 & $0,0 \mathrm{~A}$ \\
\hline testemunha & $0,0 \mathrm{~A}$ & $0,0 \mathrm{~A}$ & $0,0 \mathrm{~B}$ & 0 & $0,0 \mathrm{~B}$ & $0,0 \mathrm{~A}$ & 0 & $0,0 \mathrm{~A}$ \\
\hline F. blocos & $1,00^{\mathrm{NS}}$ & $0,55^{\mathrm{NS}}$ & $1,00^{\mathrm{NS}}$ & $-\cdots$ & $1,00^{\mathrm{NS}}$ & $0,49^{\mathrm{NS}}$ & $-\cdots$ & $0,94^{\mathrm{NS}}$ \\
\hline F. trat. & $1,00^{\mathrm{NS}}$ & $0,91^{\mathrm{NS}}$ & $3,67^{* *}$ & $-\cdots$ & $3,67 * *$ & $0,90^{\mathrm{NS}}$ & ---- & $1,69^{\mathrm{NS}}$ \\
\hline CV $(\%)$ & 670,82 & 498,79 & 350,04 & $-\cdots$ & 350,04 & 482,74 & ---- & 246,72 \\
\hline D M S & 3,67 & 5,27 & 3,26 & ---- & 3,26 & 3,73 & ---- & 0,57 \\
\hline
\end{tabular}

1. Dados transformados para ARC SEN $\sqrt{ }(\mathrm{X}+0,0)$; DAA $=$ dias após a aplicaçāo

NS - Não significativo pelo teste F; * significativo ao nível de $5 \%$ de probabilidade;

** significativo ao nível de $1 \%$ de probabilidade. 
TABELA 3 - Resultados da análise tecnológica de frutos de laranjeira-'Hamlin' aos 180DAA, em resposta aos tratamentos, quando aplicados no estágio de frutos com 2 a 4cm de diâmetro. Taiúva, 1998

\begin{tabular}{|c|c|c|c|c|c|c|}
\hline \multirow{2}{*}{ Tratamentos } & \multicolumn{2}{|c|}{ D iâmetro (cm) } & \multirow{2}{*}{$\% \mathrm{~S} \mathrm{u} \mathrm{co}^{1}$} & \multirow{2}{*}{$\circ$ B rix } & \multirow{2}{*}{$\begin{array}{l}\text { A cidez. } \\
\text { titulável }\end{array}$} & \multirow{2}{*}{ R a tio } \\
\hline & $90 \mathrm{DA} A$ & $180 \mathrm{D} \mathrm{A} \mathrm{A}$ & & & & \\
\hline$C-3,12$ & $\begin{array}{ll}4,6 & \mathrm{~A}\end{array}$ & 5,8 B C & $44,6 \mathrm{~A}$ & $\begin{array}{ll}9,7 & \mathrm{~A} \\
\end{array}$ & $0,83 \quad \mathrm{~A}$ & 11,4 A B \\
\hline$C-6,25$ & $4,7 \mathrm{~A}$ & 5,9 А В C & 46,9 A & $9,6 \mathrm{~A}$ & $0,78 \mathrm{~A}$ & 12,4 A B \\
\hline C $-12,5$ & $4,7 \mathrm{~A}$ & 6,0 A B & $46,5 \mathrm{~A}$ & $9,5 \mathrm{~A}$ & $0,70 \mathrm{~A}$ & $13,7 \mathrm{~A}$ \\
\hline C -25 & $4,6 \mathrm{~A}$ & 5,9 А В C & 46,2 A & $8,7 \mathrm{~A}$ & $0,73 \mathrm{~A}$ & 11,0 A B \\
\hline$C-50$ & $4,4 \mathrm{~A}$ & 5,8 B C & 44,3 A & $8,4 \mathrm{~A}$ & $0,76 \mathrm{~A}$ & 11,0 A B \\
\hline C -100 & $4,8 \mathrm{~A}$ & $6,2 \mathrm{~A}$ & $46,3 \mathrm{~A}$ & $8,5 \mathrm{~A}$ & $0,78 \mathrm{~A}$ & 10,8 A B \\
\hline $\mathrm{CA}-1,06$ & $\begin{array}{ll}4,8 \quad \mathrm{~A} \\
\end{array}$ & 5,9 A B C & $47,5 \mathrm{~A}$ & $8,1 \mathrm{~A}$ & $0,72 \mathrm{~A}$ & 11,4 A B \\
\hline $\mathrm{CA}-3,12$ & $4,8 \mathrm{~A}$ & 6,1 A B & 45,7 A & $9,0 \mathrm{~A}$ & $0,76 \mathrm{~A}$ & 12,3 A B \\
\hline $\mathrm{CA}-6,25$ & $4,6 \mathrm{~A}$ & 5,9 А В C & $47,7 \mathrm{~A}$ & $9,6 \mathrm{~A}$ & $0,82 \mathrm{~A}$ & 11,6 A B \\
\hline C A $-12,5$ & $4,7 \mathrm{~A}$ & 6,0 A B C & 47,1 A & $9,3 \mathrm{~A}$ & $0,80 \quad \mathrm{~A}$ & 11,7 A B \\
\hline $\mathrm{CA}-25$ & 4,8 A & 6,2 A B C & $46,8 \mathrm{~A}$ & $9,3 \mathrm{~A}$ & $0,73 \mathrm{~A}$ & 12,8 A B \\
\hline $\mathrm{CA}-50$ & $4,4 \mathrm{~A}$ & $5,6 \mathrm{C}$ & $47,4 \mathrm{~A}$ & $9,2 \mathrm{~A}$ & $0,82 \mathrm{~A}$ & 11,2 A B \\
\hline C A -100 & $4,4 \mathrm{~A}$ & 5,9 А В C & $47,4 \mathrm{~A}$ & $8,8 \mathrm{~A}$ & $0,80 \quad \mathrm{~A}$ & 11,1 A B \\
\hline $\mathrm{C} \mathrm{M}$ & $\begin{array}{ll}4,5 & \mathrm{~A}\end{array}$ & 5,7 B C & $46,8 \mathrm{~A}$ & $9,6 \mathrm{~A}$ & $0,79 \mathrm{~A}$ & 12,2 A B \\
\hline testem un a & $4,6 \mathrm{~A}$ & 5,8 A B C & $47,3 \mathrm{~A}$ & $7,5 \mathrm{~A}$ & $0,79 \mathrm{~A}$ & $9,5 \mathrm{~B}$ \\
\hline F. b 1ocos & $0,73^{\mathrm{NS}}$ & $0,21^{\mathrm{NS}}$ & $3,62 *$ & $29,59 * *$ & $1,55^{\mathrm{NS}}$ & $18,39 * *$ \\
\hline F. Trat. & $2,80 * *$ & $4,83 * *$ & $1,17^{\mathrm{N} \mathrm{S}}$ & $1,66^{\mathrm{NS}}$ & $1,24^{\mathrm{N} \mathrm{S}}$ & $1,66^{\mathrm{NS}}$ \\
\hline C V $(\%)$ & 3,28 & 2,38 & 3,52 & 9,53 & 8,20 & 11,30 \\
\hline D M S & 0,46 & 0,42 & 4,96 & 2,59 & 0,19 & 3,97 \\
\hline
\end{tabular}

1. Dados transformados para ARC SEN $\sqrt{ }(\mathrm{X}+0,0)$; DAA $=$ dias após a aplicação

NS - Não significativo pelo teste F; * significativo ao nível de $5 \%$ de probabilidade;

** significativo ao nível de $1 \%$ de probabilidade.
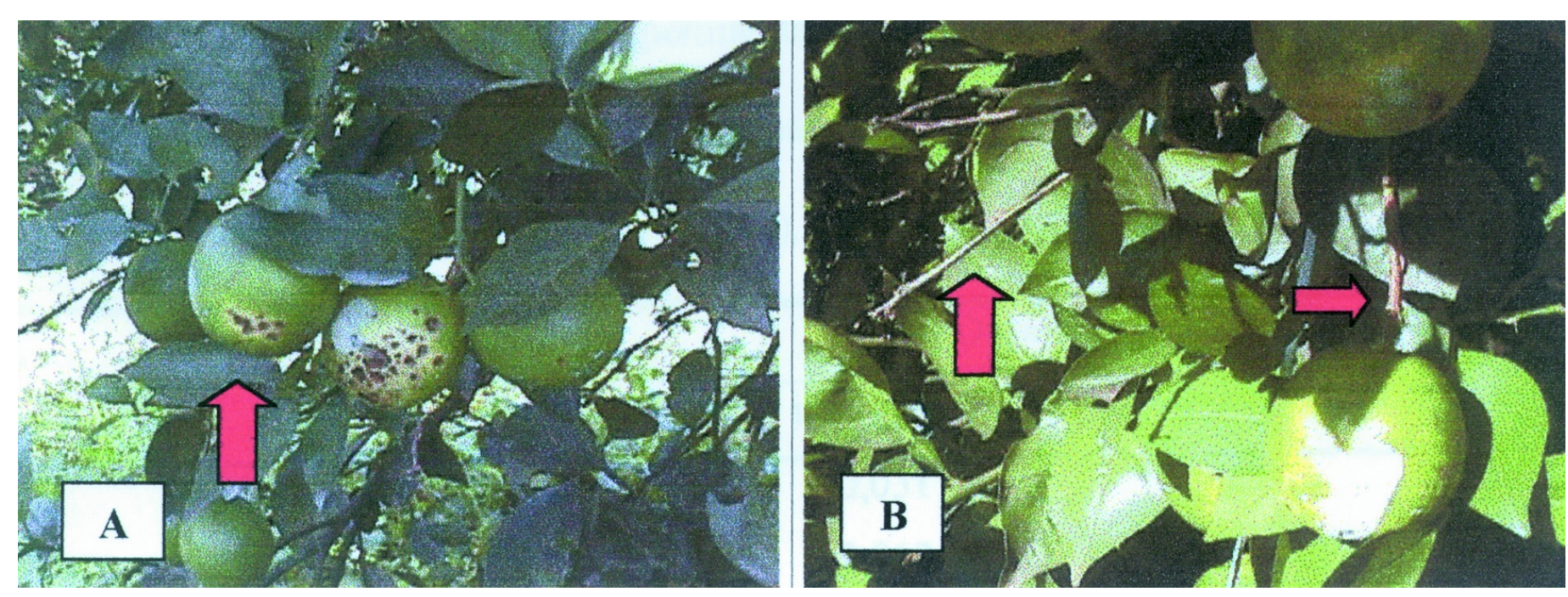

FIGURA 1 - Detalhes dos sintomas de intoxicação pela mistura de clomazone com ametryne em frutos de laranjeira-Hamlin aos 100 DAA (A), e detalhe de ramos secos (B). Taiúva-SP, 1998.

ramos, as folhas apresentaram-se totalmente cloróticas, e decorridos 60 a 90 DAA caíram ou entraram em processo de senescência, secando por completo após os 100 DAA (Figura 01-B). Segundo a FMC (s.d.) e Rodrigues \& Almeida (1998), o clomazone inibe a síntese de compostos isoprenóides, que são os precursores de pigmentos fotossintéticos, causando redução no nível de caroteno e fitol e, conseqüentemente, clorofila. Uma vez que o caroteno protege a clorofila da destruição pela luz solar, o mecanismo de ação do produto torna-se bidirecional, inibindo a produção de clorofila e a produção de pigmentos protetores da mesma. Com isso, as folhas ficam descoloridas, por falta de clorofila, secando em pouco tempo.

A absorção do produto ocorre preferencialmente pelo meristema apical da planta e, uma vez que sua translocação ocorre pelo xilema, seguindo o fluxo transpiracional, os sintomas de sua intoxicação em plantas deverá manifestar-se nos órgãos mais novos (Rodrigues \& Almeida, 1998). Contudo, os sintomas de intoxicação pelo clomazone e a mistura que se manifestaram nos 
frutos, através de manchas cloróticas e/ou necróticas, causando a mortalidade de ramos, não haviam ainda sido relatados. Também, não foi encontrado na literatura qualquer trabalho que relatasse os efeitos da deriva do clomazone, simulada ou não, sobre a porcentagem de abortamento de frutos de laranjeira, assim como sobre as características qualitativas através de análise tecnológica dos frutos.

\section{CONCLUSÕES}

A simulação de deriva de clomazone, isolado ou em mistura e na formulação microencapsulada, até as suas doses comerciais, não acarretou diferenças significativas quanto ao diâmetro dos frutos. A dose comercial de clomazone isolado e quando em mistura com ametryne a 50 e $100 \%$ da dose levou a formação de manchas cloróticas e/ou necróticas na casca do fruto e apenas quando em mistura com ametryne nas mesmas proporções, à seca de ramos que se encontravam em crescimento vegetativo. A análise tecnológica dos frutos não apresentou diferenças significativas para os parâmetros analisados.

\section{REFERÊNCIAS BIBLIOGRÁFICAS}

AUCH, D. E., ARNOLD, W. E. Dicamba use and injury on soybeans (Glycine max) in South Dakota. Weed Science, Champaign, v.26, p.471-5, 1978.
BAYLEY, J. A., KAPUSTA, G. Soybean (Glycine max) tolerance to simulated drift of nicosulfuron and primisulfuron. Weed Technology, Champaign, v. 7, n. 3, p. 740-5, 1993.

DURIGAN, J. C. Comportamento de herbicidas no ambiente In: SEMINÁRIO TÉCNICO SOBREPLANTAS DANINHASEOUSO DE HERBICIDAS EM REFLORESTAMENTO, 1989, Rio de Janeiro. Anais... Rio de Janeiro : SBS/ABRACAVE/SIF, 1989.

FMC. Manual de produtos. São Paulo, s.d. p 67-75, 83-87.

GELMINI, G. A. Herbicidas: indicações básicas. Campinas: Fundação Cargill, 1988. 334 p.

MATUO, T. Técnicas de aplicação de defensivos agrícolas. Jaboticaal: FUNEP, 1990. 136p.

RODRIGUES, B. N., ALMEIDA, F. S. de. Guia de herbicidas. 4. ed. Edição dos Autores, 1998. 648p.

TRESSLER, D. K.; JOSLYN, M. A. Fruits and vegetable juice: processing technology. Westport: The AVI Pub., 1961. 1028p.

YATES, W. E.; AKESSON, N. B., BAYER, D. E. Drift of glyphosate sprays applied with aerial and ground equipment. Weed Science, Champaign, v. 26, n. 6, p. 597-604, 1978. 